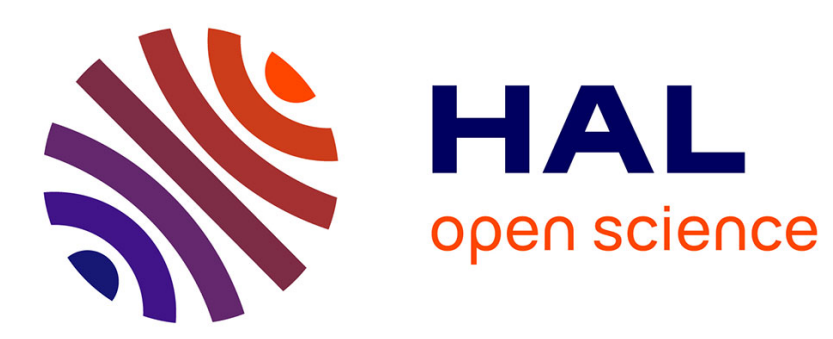

\title{
Communication et confiance au secours de la refonte du système d'information d'une entreprise de services
}

\author{
Hachimi Abba, Sylvie Leleu-Merviel
}

\section{To cite this version:}

Hachimi Abba, Sylvie Leleu-Merviel. Communication et confiance au secours de la refonte du système d'information d'une entreprise de services. Les Cahiers du numérique, 2010, Piloter l'entreprise à l'ère du numérique, 6 (4), pp.77 - 110. 10.3166/lcn.6.4.77-110 . hal-03186210

\section{HAL Id: hal-03186210 \\ https://hal.science/hal-03186210}

Submitted on 30 Mar 2021

HAL is a multi-disciplinary open access archive for the deposit and dissemination of scientific research documents, whether they are published or not. The documents may come from teaching and research institutions in France or abroad, or from public or private research centers.
L'archive ouverte pluridisciplinaire HAL, est destinée au dépôt et à la diffusion de documents scientifiques de niveau recherche, publiés ou non, émanant des établissements d'enseignement et de recherche français ou étrangers, des laboratoires publics ou privés. 


\title{
COMMUNICATION ET CONFIANCE AU SECOURS DE LA REFONTE DU SYSTĖME D'INFORMATION D'UNE ENTREPRISE DE SERVICES
}

\author{
Hachimi Abba, Sylvie Leleu-Merviel
}

Lavoisier | «Les Cahiers du numérique »

2010/4 Vol. 6 | pages 77 à 110

ISSN 1622-1494

ISBN 9782746232662

Article disponible en ligne à l'adresse :

https://www.cairn.info/revue-les-cahiers-du-numerique-2010-4-page-77.htm

Distribution électronique Cairn.info pour Lavoisier.

(C) Lavoisier. Tous droits réservés pour tous pays.

La reproduction ou représentation de cet article, notamment par photocopie, n'est autorisée que dans les limites des conditions générales d'utilisation du site ou, le cas échéant, des conditions générales de la licence souscrite par votre établissement. Toute autre reproduction ou représentation, en tout ou partie, sous quelque forme et de quelque manière que ce soit, est interdite sauf accord préalable et écrit de l'éditeur, en dehors des cas prévus par la législation en vigueur en France. Il est précisé que son stockage dans une base de données est également interdit. 


\title{
COMMUNICATION ET CONFIANCE AU SECOURS DE LA REFONTE DU SYSTÈME D'INFORMATION D'UNE ENTREPRISE DE SERVICES
}

\author{
HACHIMI ABBA
}

SYLVIE LELEU-MERVIEL

\begin{abstract}
La refonte du système d'information d'une entreprise a des implications organisationnelles et managériales. L'objectif du projet étudié ici est de répondre aux besoins technologiques et fonctionnels de la société. Cependant, là où les managers infèrent que le projet est abouti lorsque l'upgrade technique est accompli, il est apparu que les innovations technologiques et l'implantation de nouveaux outils ne suffiraient pas : il convenait de faire adhérer toutes les parties prenantes au projet. Le point de vue communicationnel s'est donc trouvé projeté au cœur de la problématique. Il a fait émerger l'impossibilité de considérer l'entreprise comme un bloc homogène dans le triptyque de référence Entreprise/Service numérique/Client.
\end{abstract}




\section{Introduction}

Pendant longtemps, les entreprises, pour assurer leur pérennité, ont essayé de mettre en place un mode organisationnel orienté vers la stabilité. L'efficacité du système managérial était liée à l'aptitude à rester stable définitivement. Mais les approches systémiques de l'organisation (Mélèse, 1990) montrent que l'entreprise est en interaction permanente avec son environnement.

Le changement est devenu une préoccupation majeure des entreprises, pour lesquelles l'efficacité réside désormais dans leur capacité à évoluer en continu et sans heurts. Avec l'évolution rapide des techniques, et l'émergence de (nouvelles) technologies de l'information, les systèmes d'information jusqu'alors organisés en systèmes fonctionnels (SI pour la gestion comptable et financière, SI pour la gestion de production, SI pour les RH) prennent une dimension interorganisationnelle (Reix, 2005), évoluant vers l'intégration. Des projets tous azimuts sont lancés, qui consistent à mettre en œuvre des nouvelles architectures informatiques ou refondre un système existant.

Dans le projet étudié ici, on présente comment l'impératif communicationnel interne s'est interposé entre la vision managériale et la réalité de l'interaction au travail, en vue d'obtenir une transaction coopérative impérative de la part de toutes les parties prenantes.

C'est ainsi qu'au-delà de l'aspect technique, la mise en œuvre de ce projet informatique a donc constitué, pendant tout son déroulement, un point focal de la gouvernance de cette entreprise : faire adhérer toutes les parties prenantes en accompagnant humainement l'implantation du projet, et replacer la communication avec les individus et les groupes au centre du processus managérial. On y expose les difficultés rencontrées et la solution qui a émergé : la conception et la mise en œuvre de la méthode 4C, coopération créative basée sur la communication et la confiance.

\section{De l'organisation taylorienne à l'entreprise numérique via le système d'informations}

On peut remonter très loin pour tenter d'inscrire l'organisation de l'activité humaine dans le temps. Déjà Platon, dans la République, estimait que l'on travaillait plus et mieux et plus aisément, lorsque chacun ne fait qu'une chose, celle à laquelle il est propre. L'histoire récente montre aussi l'importance de l'organisation dans les grands travaux comme ceux menés par l'ingénieur du roi Vauban (1633-1707) ou la mise en place, en 1586, de l'obélisque du Vatican (de plusieurs centaines de tonnes), pour ne citer que ces deux exemples. 
D’une manière générale, on distingue les organisations suivant trois périodes : organisations pré-tayloriennes ou prébureaucratiques, tayloriennes ou bureaucratiques, et post-tayloriennes ou post-bureaucratiques.

\section{Les premières théories $d u X X^{e}$ siècle : Taylor, Fayol, Weber}

Au regard de la littérature sur ce sujet, nous considérons le développement des théories de l'organisation à partir des débuts du XXe siècle. En effet, cette période est marquée par les travaux de l'américain F. Taylor (1856-1915) et du français H. Fayol (1841-1925). Taylor est connu pour son organisation scientifique du travail qui se base sur la division du travail. Pour Taylor qui a publié ses idées dans deux ouvrages principaux : Shop management (Taylor, 1903) et Principles of scientific management (Taylor, 1911), gagner en productivité signifie en résumé utiliser de façon maximale les outils de production, supprimer les gestes inutiles des ouvriers et assurer une préparation minutieuse du travail ainsi que son suivi. Mais l'histoire a montré que cette organisation du travail reste très limitée. Quant à Fayol, sa doctrine est bien exprimée à travers le titre de l'exposé de ses réflexions: administration industrielle et générale, prévoyance, organisation, commandement, coordination, contrôle. Henry Fayol (1916) apporte une double réponse aux questions de ses contemporains : il donne une théorie du commandement et des chefs; il propose des solutions pour gérer des organisations complexes et échapper à leurs effets pervers. Mais les critiques sur sa théorie s'expriment par rapport à la rigidité et à l'aspect contraignant de son approche au regard de la place qu'il donne à la hiérarchie.

Cependant, la vision de l'organisation en ce début du XXe siècle n'était pas seulement taylorienne ou fayolienne, elle était aussi sociologique avec Max Weber pour qui l'intérêt porte sur les fondements de l'autorité et du pouvoir dans les organisations : il s'interroge ainsi sur les raisons pour lesquelles les organisations, en particulier bureaucratiques, se sont développées (Bagla, 2003). Weber, qui est contemporain de Taylor, pense que l'organisation bureaucratique est la seule forme de domination légale que l'homme moderne est prêt à accepter ; quant à Taylor, la question de la justification de son organisation du travail ne se pose pas pour lui, puisqu'elle est censée être «scientifique». 


\section{Approches managériales et sociologiques}

Dans l'étude des organisations, on fait toujours référence aux expériences ${ }^{1}$ menées par Elton Mayo (1880-1949) réalisées peu avant la grande crise de 1929 et qui se sont déroulées dans les usines de la Western Electric à Chicago, chez les ouvrières principalement. L'intérêt de ces expériences vient du fait qu'elles démontrent que les avantages matériels ne sont pas les seules motivations au travail mais que des critères comme la reconnaissance du travail ou la qualité des relations avec la hiérarchie sont tout aussi importants. Elles marquent une rupture avec le taylorisme.

Les expériences de Chicago ont ouvert la voie à de nombreux chercheurs de diverses disciplines intéressés par le fonctionnement des organisations. Au début, les travaux ont porté en grande partie sur la motivation, avec Kurt Lewin (1946) inventeur de la «dynamique de groupe» ou de la méthodologie «recherche-action», Maslow (1943) et sa pyramide ${ }^{2}$ des besoins ou Chris Argyris (1957) dont les travaux ont porté sur les relations des individus avec leurs organisations ainsi que la conduite du changement. Mais on ne peut cantonner l'organisation dans un seul champ de recherche disciplinaire. Elle est fondamentalement pluridisciplinaire. On peut d'une part évoquer une approche «managériale » des organisations et, d'autre part, une approche sociologique. Le regard managérial des organisations exprime l'influence socio-économique à laquelle elles sont soumises. Diverses théories propres à la gestion ont été développées : théorie de la firme avec James March et Richard Cyert (1963), théories de la décision avec Herbert Simon (1959), théories des coûts de la transaction d'Olivier Williamson (1975), etc.

Les sociologues émettent des réserves sur cette approche parce qu'elle se base sur «la rationalité et la prévisibilité dans un espace clos et limité ». C'est une approche qualifiée de normative. Leur analyse organisationnelle est centrée sur les hommes et leurs actions. Herbert Simon, qui avait quant à lui une

1. Ces expériences sont connues sous le nom d'expériences de Hawthorne du nom de l'usine; elles se sont déroulées entre 1927 et 1929. L'équipe Mayo en conclut que l'estime de soi, la cohésion étaient plus importantes pour la productivité que l'état matériel de l'environnement de travail. C'est la rupture avec les hypothèses de Taylor qui étaient basées sur l'intérêt individuel. C'est aussi la naissance de l'école des relations humaines.

2. Abraham Maslow (1908-1970) classe les besoins des individus suivant une pyramide de cinq niveaux qui va des besoins physiologiques nécessaires à la survie humaine (boire, manger, etc.) aux besoins d'accomplissement de soi comme besoins les plus élevés à satisfaire. Ce modèle a été critiqué pour sa linéarité, et même sa légitimité a été contestée. 
démarche pluridisciplinaire, a étudié l'organisation administrative et la prise de décision; pour lui, «pour comprendre en quoi le comportement de l'individu s'intègre dans le comportement de l'organisation, il est nécessaire d'étudier la relation qui existe entre la motivation personnelle de l'individu et les objectifs que poursuit l'organisation » (Simon, 1983, p. 19).

L'organisation taylorienne ou bureaucratique se fonde sur le respect des règles censées garantir l'efficacité au travail et réduire les incertitudes. Les sociologues estiment plutôt que ces règles résulteraient des interactions humaines et qu'elles sont difficiles à respecter. C'est pourquoi ils proposent une distinction entre une organisation formelle et une organisation informelle. Dans l'organisation formelle, on retrouve l'organisation officielle avec des buts et des intentions précis pour orienter les interactions entre ses membres. Dans l’organisation informelle se développent les pratiques imprévues et les déviations ; ces déviations ou contournements des règles profitent le plus souvent à l'entreprise selon Bagla. Crozier et Friedberg, qui ne voient pas l'utilité de cette distinction, estiment dans L'acteur et le système (1977) que, si l'action des acteurs s'inscrit dans un contexte concret, celle-ci est sans cesse remise en cause par leurs pressions et leurs stratégies.

Les approches sociologiques se sont développées dans les années 1970. Outre Crozier et Friedberg, on peut faire référence aux travaux d'auteurs comme R. Sainsaulieu avec la Sociologie de l'organisation et de l'entreprise (1987). Après avoir exploré la dynamique des modèles de l'histoire industrielle et postindustrielle des organisations, il y examine les relations individuorganisation avec la notion de culture, d'identité et la régulation sociale de l'entreprise. D'autres auteurs (Boltanski, Salais, Callon, Latour...) ont contribué à développer ces théories; Anthony Giddens (Giddens, 1987), estime que les structures sociales ont un caractère ambigu parce qu'elles sont à la fois contraignantes pour l'acteur mais elles lui permettent aussi d'exister. Les organisations sont aussi vues sous l'angle économique (Drucker, 1993) ou dans une approche constructiviste (Bouchikhi, 1990).

\section{Une nouvelle organisation par le projet}

La littérature autour des théories de l'organisation est immense du fait même de l'objet d'étude qu'est l'organisation. Dans L'analyse des organisations: une anthologie sociologique, Séguin et Chanlat (Seguin et al, 1986) résument l'organisation sous deux visions : une vision fonctionnaliste de l'organisation et une vision critique de l'organisation. 
La vie de ces organisations est rythmée par les projets qu'elles mettent en œuvre. Le terme projet a dans le passé désigné la façon de s’organiser dans la construction des grands ouvrages, monuments ou infrastructures avec des moyens matériels et humains très importants.

Dans L'analyse de systèmes techniques avancés de management, Cleland et King définissent le projet comme «étant un effort complexe pour atteindre un objectif spécifique, devant respecter un échéancier et un budget, et qui, typiquement, franchit des frontières organisationnelles, est unique et en général non répétitif dans l'organisation» (Cleland et King, 1970). On peut le définir comme une création collective, organisée dans le temps et l'espace, en vue d'une demande.

Plus généralement, on distingue aussi des catégories de projets appelées projets durs ou hard projects qui concernent un bien livrable tangible : un pont, une autoroute, un barrage hydroélectrique; et aussi projets mous ou soft projects qui délivrent un bien nettement moins tangible: un nouveau programme de formation professionnelle, une nouvelle stratégie de communication, un nouveau système d'information, etc.

\section{La vision «système d'information »}

La terminologie «système d'information » a un tel succès de nos jours dans les entreprises et organisations qu'il peut sembler inutile de disserter sur le sujet: tout le monde en parle, chaque entreprise semble en posséder un, voire plusieurs, plus ou moins identifiés.

\section{Une redéfinition conceptuelle}

Parler de système d'information soulève toujours la nécessité de circonscrire le concept de «système » mais surtout celui «d'information » et de «donnée » Leleu-Merviel, 2008). La question est d'autant plus délicate, comme le souligne Sylvie Leleu-Merviel, que "malgré les nombreux travaux, une partie de l'information reste mystérieuse» (Leleu-Merviel, 1997, 79). La démarche de l'auteur est de «déterminer un vocabulaire opérant ». Cette démarche consiste à définir la notion de donnée, de signal, d'information, de sens et enfin de message; elle nous permettrait de mieux comprendre les interrogations autour de l'information car comme l'indique Yves Jeanneret ${ }^{3}:$ "L'ensemble du glossaire ne suffit pas à définir la notion d'information, tant est étendu le spectre de ses usages et de ses significations possibles aujourd'hui. On pointera

3. La société de l'information, glossaire critique, p. 87, La documentation française. 
seulement ici quelques-uns des problèmes que pose l'extension considérable de la notion $»$.

Concrètement, les divers points de vue sur le système d'information résultent ainsi de la manière dont chacun dans l'organisation conçoit l'information et sa finalité. Une des évidences que l'on peut constater dans les entreprises est que par exemple, les informations concernant la vie syndicale, l'information du personnel ne sont pas considérées comme faisant partie du système d'information, ce qui accrédite l'hypothèse que la référence est informatique. Le système d'information est, pour beaucoup, quelque chose de quantifiable, technique, efficace, sérieux alors que pour eux, l'information est quelque chose de qualitatif, subjectif, flou, voire conflictuel. On a donc bâti jusqu'ici, toujours selon Mélèse (1979), plutôt des systèmes de données et l'auteur note qu'il faudrait aller vers une approche de système d'information qui se centre sur la notion de situation informationnelle ${ }^{4}$ des individus ; l'information est une composante du système socio-technique indissociable de l'organisation et de sa communication. Les interactions internes et externes à l'organisation sont porteuses d'informations. Ne pas dissocier le système d'information du système socio-technique pris dans sa globalité et renoncer à connaître, saisir et codifier toute information semble être une piste, une nouvelle approche pour définir le système d'information: «le concept de système d'information désignerait alors l'ensemble interactif de toutes les situations informationnelles, autrement dit, le jeu complexe de tous les échanges d'information signifiante ».

\section{Point de vue techniciste}

Et pourtant, comme l'affirme Mélèse (1979), dans une entreprise, lorsqu'on «tente de repérer tout ce qui informe, tous les signaux, tous les messages, toutes les perceptions qui influent sur notre activité et notre comportement... et qu'on détermine ensuite la partie de l'information qui provient de ce qui est officiellement nommé système d'information puis, à l'intérieur de ce dernier, la part de ce qu'on appelle système informatique, une quantité appréciable d'information échappe au système d'information officiel et, encore bien plus, au système informatique $»$. On se demande alors si, dans beaucoup de cas, voire dans certaines disciplines qui l'étudient (management, informatique..), le

4. Selon Mélèse, «il s'agit de caractériser l'unité comme un élément de l'organisation elle-même, considéré comme un système informationnel : idéalement, il faudrait repérer les productions et échanges de significations entre l'unité d'une part, l'organisation et l'environnement d'autre part, afin dévaluer le système d'information comme l'ensemble de ces significations » (Mélèse, 1979, 30). 
système d'information ne serait pas confondu avec le ou les systèmes informatiques tout court.

Dans la vision «informaticienne» d'un système d'information, on parle : bases de données de l'entreprise, applications métiers, infrastructure réseau, serveurs de données et systèmes de stockage, serveurs d'application, postes de travail informatiques, dispositifs de sécurité, etc. l'accent est donc mis en particulier sur le dispositif technique (matériel et logiciel). On parle d'architecture des systèmes d'information dont la base principale est composée de l'ordinateur et du logiciel. Le traitement électronique de l'information sous sa forme numérisée a occulté toutes ses autres dimensions évoquées plus haut. Ces diverses techniques ou fonctions de base (de saisie, stockage, traitement, communication) conduisent (malheureusement) à assimiler système d'information à système informatique. Avec l'évolution rapide de ces techniques, on parle désormais de (nouvelles) technologies de l'information. Les systèmes d'information jusqu'alors organisés en systèmes fonctionnels (SI pour la gestion comptable et financière, SI pour la gestion de production, SI pour les $\mathrm{RH}$ ) vont prendre une dimension interorganisationnelle (Reix, 2005), évoluant vers l'intégration. Cette recherche d'intégration conduit à parler de nos jours dans le lexique informatique « d'urbanisation ${ }^{5}$ » de système d'information à l'image de la ville dont les problèmes liés à son développement peuvent s'appliquer aux SI: encombrement, coûts de maintenance élevés, modernisation etc.

Globalement, en plus de l'architecture métier (structuration du SI par les activités métiers de l'entreprise), on distingue deux principales architectures :

- une architecture applicative qui concerne la structuration des applications informatiques de l'entreprise ainsi que les messages échangés entre elles. Autrefois fédérées, ces applications sont désormais intégrées grâce (ou à cause) de l'urbanisation ;

- une architecture qui est la structuration des moyens d'infrastructures techniques : serveurs, poste de travail et autres périphériques.

L'étude de cas du projet système d'information développée plus loin nous donnera plus d'éléments de cette vision techniciste du SI.

5. Des nombreuses publications dans ce domaine (Volle et al, 2003, Morley et al, 2002) montrent le rôle vital de l'urbanisation des SI dans les stratégies des entreprises. Ces SI doivent justement être en phase avec l'évolution constante des marchés, la recherche d'une compétitivité toujours plus forte. 


\section{Une vision plus humaine et sociale}

Néanmoins, l'étude de l'organisation dans ses liens avec la technologie n'est pas nouvelle et elle a fait l'objet d'une abondante littérature en sciences humaines et sociales. L'introduction des technologies de l'information et de la communication a conduit à des profonds changements organisationnels. La mise en œuvre des projets de «système d'information »- désigné par SI dans la suite - par l'introduction massive des TIC dans les entreprises est un objet régulièrement analysé en sciences de l'information et de la communication, notamment en communication des organisations (Bouzon et al, 2005).

En sciences de l'information et de la communication, certains auteurs ${ }^{6}$ tiennent toujours à rappeler que le système d'information n'est pas un système informatique (Roux, 2004). Pour Brigitte Guyot, «un système d'information n'est pas simplement un système technique de traitement de l'information. Il mobilise avant tout un système social, des acteurs munis de compétences notamment cognitives, selon des normes organisationnelles et éventuellement techniques. Un système d'information peut alors se voir comme une forme instituée, reconnue, correspondant à une organisation dévolue à la mise en mémoire ou à l'exploitation de l'information. Il gère des représentations qu'il formate, transforme et rend disponibles » (Guyot, 2004).

\section{Le SI et les sciences de gestion}

Une autre approche du système d'information (SI) est due aux sciences de gestion qui ont constitué un champ de recherche en leur sein (Rowe, 2002). Les chercheurs de cette discipline se sont beaucoup plus intéressés à la contribution des SI dans l'amélioration de la performance de l'organisation. On distingue un courant ingénierique dont la préoccupation est de construire un «bon système d'information»; c'est un courant de recherche centré sur l'amélioration du processus de prise de décision en termes d'efficience, de satisfaction et d'efficacité. Selon les auteurs, malgré la diversité des modèles utilisés (normatif, impact organisationnel), les niveaux d'analyse (individus, groupe, organisation, réseau, industrie), la multiplicité des mesures (efficacité, efficience, productivité, satisfaction, flexibilité etc.), «l'orientation générale demeure et positionne très clairement le champ des SI à l'intérieur des sciences de gestion ». Cependant, ils admettent que l'objet initial de la recherche est l'information et que celle-ci doit être définie de manière indépendante de la technologie; ce qui n'est pas le cas en sciences de gestion où la recherche en SI a été « continuellement poussée par l'innovation technologique ».

6. Le Moigne, 1973 ; B. Guyot, 2004. 


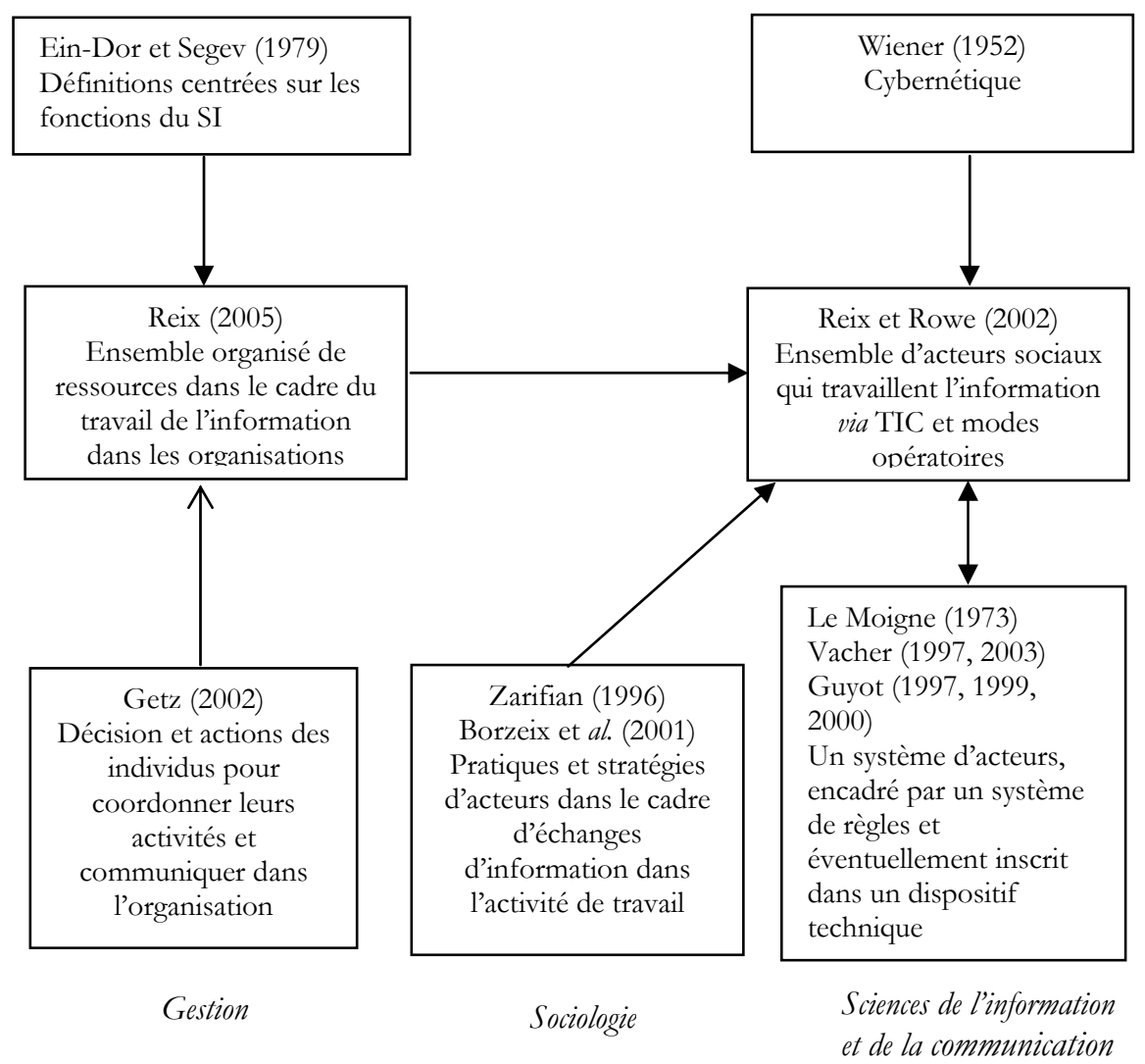

Figure 1. Les approches du SI selon 3 disciplines et points de convergence (Roux, 2004)

En sciences de gestion, l'émergence du champ de recherche sur les SI date du milieu des années 1960, avec plusieurs objets (technologie, information, connaissance). Toujours selon Rowe, «le résultat actuel est une grande diversité apparente où l'image du champ ressemble plutôt à celle d'îlots de connaissance relativement isolés et non celle d'une construction solidement organisée autour d'un paradigme unificateur ». Les auteurs clairement identifiés dans la discipline (Reix, 2005; Alter, 1999 ; Hirscheim et al., 1995 ; Mason et al., 1973) donnent des définitions diverses du SI. À cela, il faut ajouter la vision anglo-saxonne du système d'information (Ein-Dor et al., 1993) ou une vision (européenne) par le biais des individus (Getz, 1994).

Ainsi à côté de la définition de Jacques Mélèse qui considère le SI comme «un ensemble de signification», G. Davis, M. Olson, R. Ajenstat et J.L. 
Peaucelle l'identifient à un "système utilisateur-machine intégré qui produit l'information pour assister les êtres humains dans les fonctions d'exécution de gestion et de prise de décisions ». On peut donc caractériser le SI à travers sa dimension conceptuelle, technique ou pragmatique. Dans ces dimensions interfèrent des problématiques de contenu (les interactions au sein du système) et de contenant (appareillages technologiques). Les sciences de gestion admettent en définitive que la nécessité d'une réflexion sur l'ontologie du système d'information doit conduire à une proposition de définition qui dépasse la discipline elle-même, et qui affirme l'essence humaine et sociale du SI : « un système d'information est un ensemble d'acteurs sociaux qui mémorisent et transforment des représentations via des technologies de l'information et des modes opératoires ». La notion d'acteur social dans le SI indique qu'il agit et interagit avec d'autres acteurs, donc stratégiquement (Crozier et al., 1977).

\section{Bilan}

Il semble difficile de trouver une définition consensuelle du système d'information et nous préférons en guise de conclusion présenter les liens qui peuvent exister entre les différentes approches en nous inspirant de la synthèse de (Roux, 2004).

\section{Le projet de système d'information Pi.com}

\section{Contexte du projet}

La convergence informatique est une initiative lancée par l'AGIRC (Association générale des institutions de retraite des cadres) et de l'ARRCO (Association des régimes de retraites complémentaires), fédérations des institutions de retraite complémentaire des salariés du privé. Ce programme de convergence informatique a pour objectif de bâtir communautairement un système d'information unifié qui serait utilisé par les institutions de retraite complémentaire du monde AGIRC-ARRCO. Les fonctions d'interlocution ${ }^{7}$ client et d'exploitation sont sous la responsabilité de chacune des institutions. Il

7. L'interlocution client consiste à (re) placer le client au cœur de la stratégie de l'entreprise et surtout de son système d'information. Concernant l'IRCEM l'interlocution client devrait permettre au groupe de connaitre ses clients afin de leur proposer des produits adaptés et leur rendre les services attendus. Cette démarche contribuerait «à la simplification des démarches administratives de tout adhérent du groupe, an développement de l'image du groupe, à augmenter la productivité, etc.» (Source: note de lancement du projet). 
s'agit précisément pour les institutions de retraite et prévoyance comme l'IRCEM de :

- dissocier ce qui est « cœur métier » de la retraite des autres activités des caisses de retraite (prévoyance, mutuelle...) ;

- disposer des mêmes applications cœur métier pour tous les groupes ;

- mettre en commun des briques fonctionnelles qui constituent l'Usine Retraite (UR) ;

- chaque groupe a la charge d'insérer l'UR dans son environnement.

À l'origine du projet, une étude d'urbanisation a été menée au terme de laquelle le système cible a été découpé en plusieurs grandes briques fonctionnelles: le recouvrement des cotisations, le service transverse, le paiement des allocations, la gestion des adhérents, la gestion des droits, et la déclaration nominative. Pour chaque domaine, un acteur éditeur a été retenu.

L'IRCEM est sous la tutelle de la fédération AGIRC-ARCCO, organisme garant de l'équilibre des régimes de retraite complémentaire. C’est aussi la première institution de retraite complémentaire ARRCO en nombre de comptes gérés (employés, salariés, retraités), soit 4,7 millions de comptes actifs en 2002.

\section{Bref bistorique du projet}

En 2002, une réflexion en profondeur sur la stratégie de la société pour les années à venir conduit l'IRCEM à remettre en cause son système d'information.

Le projet débute réellement en 2003 et couvre toute la partie qu'on appelle «prévoyance», à savoir le traitement des dossiers de paiement des indemnisations d'arrêt de travail et toutes activités associées. Le groupe gère en effet plus de six millions d'assurés dont un million en prévoyance sur ce qu'on appelle des risques lourds : indemnités journalières, incapacité, invalidité, capital décès, etc. Avec plus de 53000 demandes d'indemnités journalières par an, les anciennes infrastructures ne pouvaient supporter la montée en charge de l'activité de la prévoyance.

Baptisé «Pi.com», le projet a pour vocation de remplacer un système existant nommé « Gaspard » qui ne répond plus aux besoins technologiques et fonctionnels de la société : Gaspard est ce qu'on appelle une application métier dans le langage informaticien, c'est-à-dire qu'elle décrit les opérations qui sont faites sur les données en fonction des requêtes des utilisateurs, ces données étant elles-mêmes sur une base. La figure 2 donne une image (très simplifiée) de son fonctionnement. 


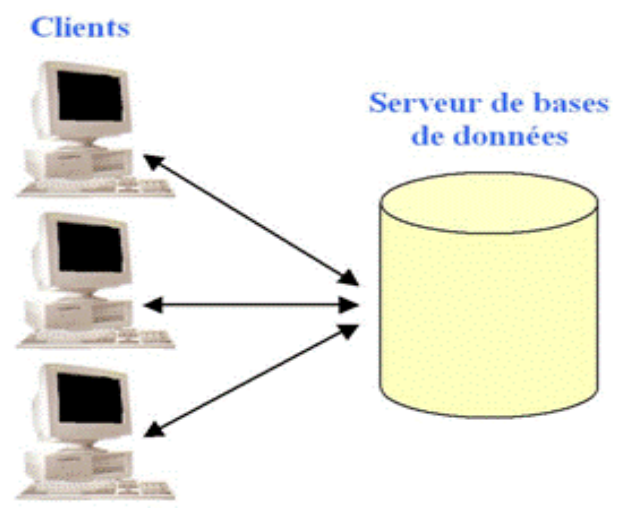

Figure 2. L'existant du système d'information (avant la refonte)

La refonte du système existant a pour buts techniques :

- d'automatiser les processus métiers ;

- d'ouvrir le système aux employés, associations et partenaires institutionnels ;

- de moderniser et pérenniser le SI par le passage à une nouvelle architecture.

Cette architecture montre que le projet Pi.com est un ensemble de sousprojets qui correspondent à un découpage en métadomaines, c'est-à-dire des grands sous-ensembles: Pythagore (gestion), Hermès (commercial), Aphrodite (clients), Euclide (comptabilité) le tout basé sur une architecture technique de développement Archimède.

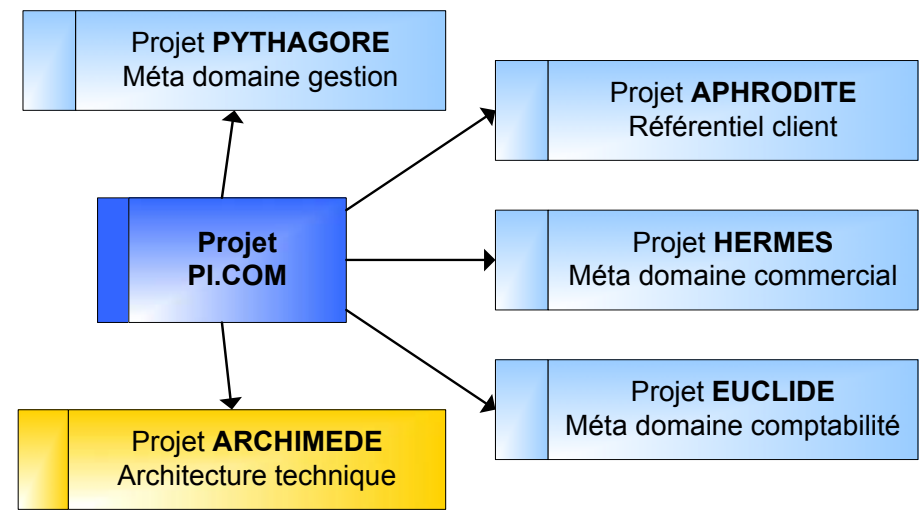

Figure 3. Vue simplifiée de la constitution de Pi.com 
Derrière cette organisation technique se nouent des échanges et des interactions entre différentes catégories d'acteurs du projet. La dimension et le contexte du projet, font qu'il implique des acteurs internes et externes à l'organisation.

\section{Pi.com : projet informatique, système d'acteurs}

Tout projet informatique est sous-tendu par un système de rapport entre acteurs. Ces acteurs sont issus des groupes différents: utilisateurs, informaticiens, maître d'ouvrage, maitre d'œuvre, fournisseurs... Cet ensemble est le lieu d'interactions et de jeux complexes et fait la particularité des projets informatiques. Selon Marciniak et Rowe, «l'examen de ces particularités conduit à souligner les aspects du management de projet qui s'avèrent pertinents pour ce type de projet : l'analyse des risques, la préparation du projet, la gestion des conflits et du système d'acteurs » (Marciniak et al., 2005).

La question de l'analyse du risque est essentielle même si nous ne la traitons pas dans cet article, tant elle est vaste et surtout particulière aux projets informatiques: de nombreuses études montrent que depuis plus d'une trentaine d'années les projets d'implantation de technologies de l'information connaissent des taux d'échec élevés, malgré les importantes ressources qui y sont allouées ${ }^{8}$. Selon Kappelman et McLean, une des causes évidentes de cette situation vient $\mathrm{du}$ fait que les groupes d'acteurs ne parlent pas le plus souvent le même langage : alors que les utilisateurs ou usagers de système informatique parlent de sa capacité à satisfaire leurs besoins en information, de sa convivialité, les spécialistes (informaticiens) voient plutôt la performance technique du système, sa facilité de maintenance ou l'absence d'erreurs (Kappelman et al, 1994).

L'aspect qui nous semble particulièrement important est la gestion du système d'acteurs et éventuellement des conflits. En théorie, chacun joue une partition dans le projet. Par exemple, la maitrise d'ouvrage (MOA), qui est représentée généralement par un ou plusieurs départements métier (ou la direction générale), se charge d'élaborer en amont un cahier des charges. C'est un document qui spécifie les besoins fonctionnels de l'application à développer. Il est généralement réalisé en lien avec la direction des systèmes d'information

8. La première véritable étude sur la difficulté des projets informatiques est celle de Frederick Brooks à travers l'ouvrage The Mythical Man-Mont: Essays on Software Engineering (1975) où pour endiguer la dérive coûteuse du projet de système d'exploitation, IBM a cru bon d'ajouter d'autres personnes puisque le projet était estimé en jours-hommes, ce qui ne fit qu'empirer la situation. Il en est de même dans les projets multimédias (Durand et al., 1997 ; Huart et al., 2000). 
(DSI) qui apporte ses connaissances de l'environnement technique et son expérience des solutions technologiques, en vue de juger de la faisabilité des demandes. La maîtrise d'œuvre (MOE) - qui couvre développement et intégration - est prise en charge par la DSI. Elle peut également être soustraitée (entièrement ou en partie) à une ou des sociétés de services (fournisseurs) etc. Concernant Pi.com, les acteurs du projet sont :

- la maitrise d'ouvrage (MOA), assurée par la direction générale avec les départements métiers de la prévoyance (dans lesquels se trouvent les utilisateurs);

- la maitrise d'œuvre (MOE) qui relève de la DSI ; à noter ici qu'une autre catégorie d'acteurs entrent en action, notamment des équipes informatiques externes ou prestataires de services qui sont plus nombreux que les internes ;

- le conseil d'administration pour le caractère stratégique du projet ;

- l'unité Recherche et Développement, entité composée de chercheurs.

On ne peut pas faire une analogie parfaite par rapport à la catégorisation de Boutinet, mais la situation des chercheurs de la R\&D par exemple, les place dans une position d'acteurs confrontants : la critique sur la faible prise en compte dans le projet de l'aspect sécurité des applications par l'un des chercheurs, spécialisé dans le domaine, a permis une évolution significative de la démarche projet; cependant, sans être conflictuels, les rapports entre l'équipe MOE et l'entité R\&D ne les ont pas incités à la coopération.

Le projet Pi.com nous conduits à analyser d'une part des situations de communication entre les informaticiens eux-mêmes et, d'autre part, entre les informaticiens et la maitrise d'ouvrage en particulier les utilisateurs futurs du projet.

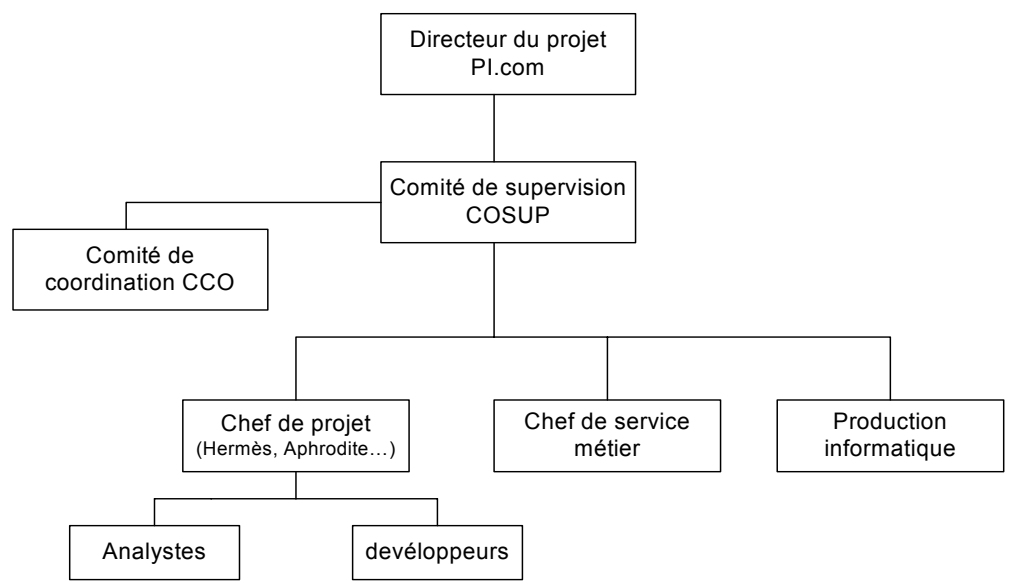

Figure 4. Les acteurs directs de Pi.com 


\section{Relations informaticiens-informaticiens}

Beaucoup d'organisations sont passées à l'externalisation de leur service informatique, espérant réduire les coûts liés aux investissements dans les technologies de l'information. Le recours à des sociétés de services et d'ingénierie informatiques (SSII) pour les projets, même de petite taille, semble être la règle. Ce qui pose la question de la sous-traitance de l'informatique des entreprises. Beaucoup d'auteurs ayant travaillé sur le phénomène (Feeny et al., 2000) estiment que la sous-traitance générale et uniforme s'avère être une mauvaise décision, une erreur qui peut coûter cher; ne pas sous-traiter en est une autre. Une sous-traitance sélective serait une piste pour les entreprises.

La MOE, la direction de système d'information en l'occurrence a fait appel à plusieurs SSII dans le cadre de Pi.com pour développer les sous-ensembles indiqués plus haut. Les «externes» représentaient $90 \%$ des informaticiens engagés sur le projet, ce qui n'est pas sans risque pour le directeur de système d'information ${ }^{9}$ (DSI). Ils sont "recrutés» en fonction de la demande de ressources dans les projets et de leurs profils métiers ${ }^{10}$ (développeurs, analystes, chefs de projet MOE, chef de projet MOA, chefs de projet junior, etc.). Plusieurs questions résultent de cette configuration de projet où les acteurs sont des externes :

- les problèmes d'harmonisation de volumes horaires hebdomadaires et surtout de procédures de travail ; la gestion n'est pas seulement administrative car ces acteurs ont les contraintes de leur entreprise d'origine (35 heures par exemple) et doivent respecter celles de l'entreprise d'accueil ;

- la diversité même des acteurs; contrairement à une idée répandue, la «confrérie » des informaticiens est d'une grande diversité qui est souvent liée aux technologies sur lesquelles ils sont compétents ;

- la question de la légitimité des internes par rapport aux externes; cette légitimité est liée aux compétences techniques: plus l'informaticien est techniquement « bon », plus il est respecté par ses pairs ;

9. Avant les années 1980, on parlait de directeur informatique (appellation moins prestigieuse que DSI). L'appellation DSI est récente et, au début, la fonction était considérée comme une voie de garage, car on ne savait pas à quoi correspondait exactement un DSI. Aujourd'hui les entreprises attendent du DSI qu'il soit " visionnaire, bâtisseur, réformateur, conducteur de changement, etc. »!

10. Les fonctions de l'informatique regroupent une nébuleuse d'une trentaine de métiers selon le Club informatique des grandes entreprises de France (CIGREF) en 2003. Ces métiers sont sans cesse réactualisés, souvent nés avec la technologie en vogue. 
- la gestion de compétences des acteurs : en effet ces informaticiens, après avoir « rempli » leur contrat, partent avec leurs compétences, leurs acquis et leur expérience du projet.

C'est l'ensemble de ces défis que doit gérer le DSI. Au début, ce DSI est en réalité un directeur de projet. Il a en charge le projet et il rend compte à la direction et aux réunions du conseil d'administration. Il a recruté un chef de projet «architecte technique» pour ce projet et qui serait susceptible de manager tous les profils entrants au cours du projet.

Pour mieux communiquer, une organisation a été mise en place sous forme de comité de supervision (MOA/MOE) et comité de coordination interne à la maîtrise d'œuvre. L'objectif de cette organisation est d'être efficace pour éviter les pathologies récurrentes aux projets informatiques: dépassement de délais, dépassement de coûts, usine à gaz, projet sans pilote, spécifications floues...

Vue de l'extérieur, l'équipe informatique est soudée et se fédère autour du directeur du projet. Mais à l'intérieur, comme dans tous les projets, se nouent des relations de pouvoir qui stabiliseront le projet par l'émergence d'un leader charismatique (Boutinet, 2006) : le directeur du projet, sans doute assailli par les justifications de coûts et délais par la DG, le CA ainsi que la gestion administrative du projet, semble "perdre pied» techniquement; l'architecte technique recruté pour le projet semble, au fil de son expérience technique, acquérir une plus grande légitimité auprès des informaticiens externes. Conséquence: une fronde des développeurs et chefs de projet pour inciter l'architecte à « prendre les rennes du projet».

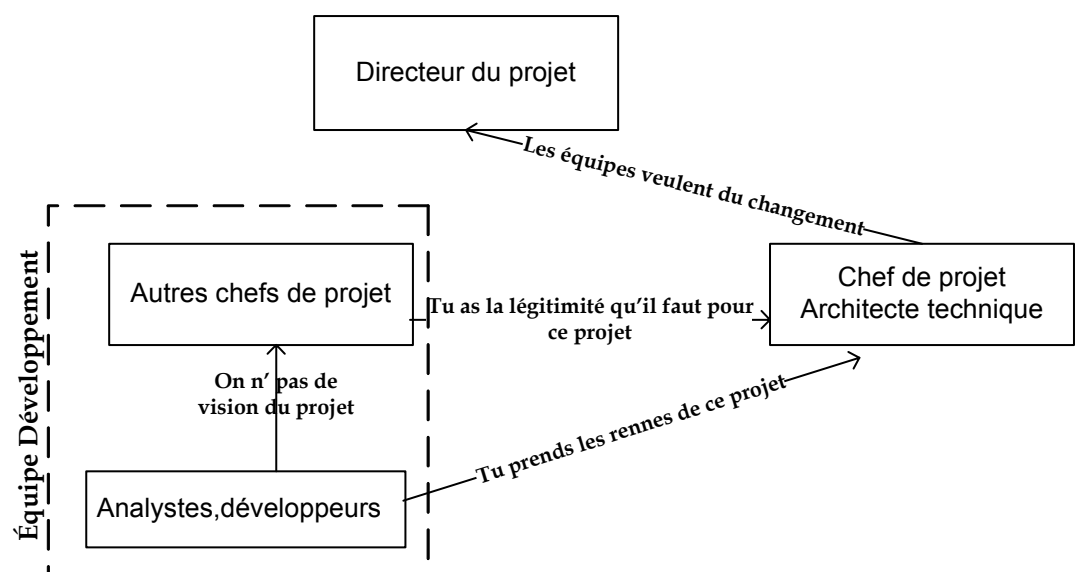

Figure 5. Relations au sein de l'équipe d'informaticiens au début du projet 


\section{Relations informaticiens-utilisateurs}

Le débat sur les réactions des salariés face aux technologies continue à faire l'objet d'une abondante littérature (Markus, 2000 ; Dorrer, 2004) ; Quan, 2006). Ces réactions viendraient du fait que l'opposition au changement est une caractéristique du comportement humain. Outre cette référence à la nature humaine (Leleu-Merviel, 2008), on peut se poser quelques questions simples sur l'introduction des technologies informatiques, par exemple, dans l'entreprise :

- les individus ont-ils leur mot à dire sur le choix de la technologie et son introduction? Un concept devenu à la mode est celui de la gouvernance des technologies de l'information IT gowvernance mais la réalité est toute autre ;

- la technologie elle-même est-elle facile à maitriser ? Est-elle adaptée aux utilisateurs?

- la formation et l'aide sont elles fournies?

- comment la communication se fait-elle autour de cette nouveauté ?

Cette dernière question nous a amené à observer les relations d'échange entre les concepteurs réalisateurs de Pi.com et les salariés qui l'utiliseront pour leur travail. Il s'agit des échanges qui vont de l'élaboration du cahier des charges à la mise en service du « produit ».

Le comité de supervision indiqué plus haut a pour rôle de permettre une plus grande coopération entre les futurs utilisateurs du SI et les informaticiens chargés de le mettre en œuvre. La tendance dans les projets informatiques est d'associer les utilisateurs, voire de créer des laboratoires tests utilisateurs. Selon Quan, "pour être sûr que les utilisateurs et les informaticiens se comprennent, on a même parfois poussé le raisonnement jusqu'à former ces mêmes utilisateurs aux méthodes Merise, RUP ou au modèles UML pour que ceux-ci puissent lire et donc valider les schémas et documents élaborés par les concepteurs informaticiens». La démarche, même si elle paraît louable et pragmatique, pose néanmoins le problème de ce que nous appellerons la compétence supplémentaire de l'utilisateur: s'il est vrai que les utilisateurs sont choisis parce qu'ils connaissent leur métier et donc savent en théorie ce que l'application doit faire, ont-ils vraiment la capacité d'exprimer leurs besoins efficacement? Cette question va se poser lors de l'élaboration du cahier des charges.

Dans le projet Pi.com, c'est la maitrise d'ouvrage (MOA) qui a la charge d'élaborer avec l'aide de la maîtrise d'œuvre le cahier des charges. Cette MOA 
est composée de la direction générale ${ }^{11}$, et des acteurs métiers (chefs de service, responsables d'unités, gestionnaires de dossiers). Au début la communication a été laborieuse.

La première difficulté entre informaticiens et utilisateurs est celle de la disponibilité des acteurs métiers pour élaborer ce cahier des charges et les spécifications qui en découlent. La MOE prend l'initiative de l'élaborer ellemême : conséquence, le cahier des charges ainsi que les réalisations qui ont été faites par la suite ne sont pas acceptées par les acteurs métiers qui estiment n’avoir pas été associés. Outre ce manque de disponibilité, les informaticiens estiment qu'il y a un déphasage entre les acteurs métiers eux-mêmes : d'une part il y a un déphasage, pensent-ils, entre la «hiérarchie du service et les subordonnés ", ces derniers ayant une toute autre vision de l'application à réaliser; d'autre part, nous passons notre temps à leur expliquer les relations interservices, ça nous prend un temps énorme et c'est exaspérant pour un informaticien à cause du temps énorme passé en $M O A$ ou assistant $M O A$. Enfin les responsables métiers n'hésitent pas à exprimer leurs inquiétudes sur l'automatisation des fonctions : attention à ne pas tout automatiser, sinon après je fais quoi moi avec mes gars.

Les relations ainsi observées au début du projet sont présentées dans la figure 6 .

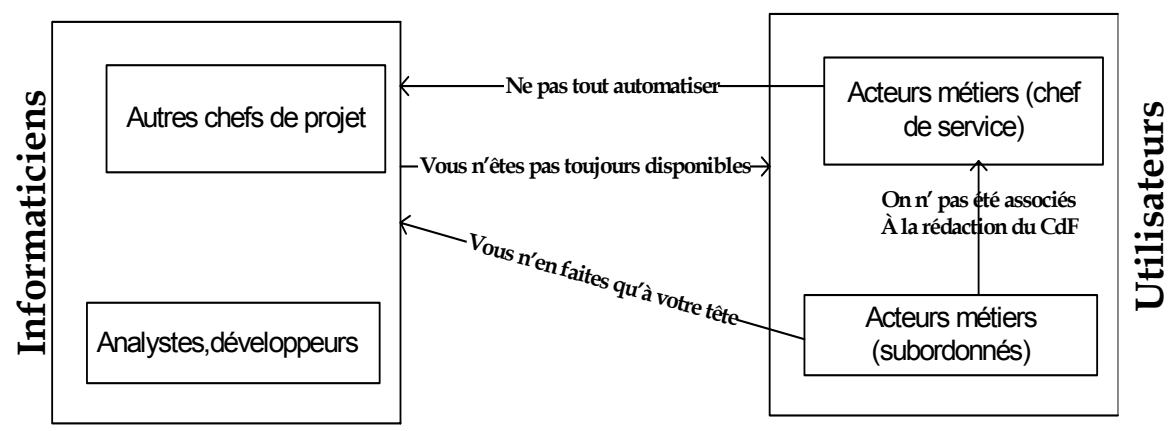

Figure 6. Relations informaticiens-utilisateurs au début du projet

Les systèmes informatiques appelés abusivement système d'information ne sont pas seulement des outils techniques au service d'une organisation. Il s'agit d'un cas particulier de projet que la composante humaine vient complexifier.

11. À l'image d'autres projets-phares comme la certification ISO, le projet Pi.com, l'informatique du groupe en général, est le domaine réservé du Directeur général adjoint. 
S'il est possible d'apporter des solutions aux difficultés techniques de mise en œuvre des systèmes d'information, la question du facteur humain demeurera posée tant que l'accent ne sera pas mis sur la communication entre les concepteurs (informaticiens) de ce «système» et les potentiels utilisateurs (salariés et autres non informaticiens).

Sans généraliser à partir de cette étude de cas, on peut conclure que les salariés sont les moins mis en avant dans les processus communicationnels des projets tels que les conçoivent ces entreprises.

Pourtant, la mise en place d'un système de communication-coopération peut amener à intégrer des variables comme la confiance qui peuvent contribuer de façon significative à la créativité de ces acteurs projet. Un modèle de travail coopératif, axé sur la communication, est envisageable pour émuler cette créativité.

\section{La communication et la confiance pour la réussite du projet}

L'approche par modélisation des relations (Mucchielli, 2004), nous montre d'une part les intrigues qui se déroulent autour du projet quel qu'il soit, d'autre part, des situations de communication parfois bloquées. Le rôle de l'être humain communicant est décisif: la compréhension des processus communicationnels est incontournable dans ce projet. Pourquoi cette composante humaine se rebelle-t-elle lorsqu'on cherche à exploiter tous ces outils formels et formalisants (outils informatiques, système d'information)? Comment dans le cadre du projet de refonte du système d'information, les freins se sont-ils "desserrés », pour arriver à un épilogue heureux qui est le lancement de Pi.com ? Comment amener les informaticiens et les utilisateurs à coopérer? Plus globalement comment créer par la communication une dynamique de la confiance dans l'entreprise pour que la gouvernance de l'entreprise ne demeure pas simplement une illusion du management (Le Goff, 2000) ? Ce climat de communication-confiance-coopération pourrait conduire naturellement chaque acteur du projet à libérer par son engagement son "processus créatif».

\section{Approche conceptuelle de la confiance}

Niklas Luhmann considère «la confiance au sens large du terme comme constituant une donnée élémentaire de la vie en société » et aussi comme un mécanisme pour réduire la complexité sociale (Luhmann, 2006). 
La confiance suscite un vif renouveau conceptuel, parce que dans un cadre général d'incertitude grandissante, il y a un accroissement de la coopération entre les individus dans les organisations. Plus généralement la question de la confiance est devenue centrale dans le pilotage des entreprises; l'actualité de France Telecom, par exemple, montre bien la «crise de confiance » vécue entre collaborateurs et Direction dans le cadre de projet d'entreprise (Reynaud et al, 2010).

La confiance, comme l'explique Jean-Louis Le Moigne ${ }^{12}$, «c'est un concept qui, s'il est merveilleux dans son usage courant, "J'ai confiance", "Tu n'as pas confiance", nous échappe dès qu'on veut chercher à le cerner et le définir ». Selon lui, le terme complexe de confiance est à la fois multidimensionnel, récursif et téléologique : il renferme bien des dimensions de connotations et n'est pas réductible à une composition; il est récursif parce qu'il désigne à la fois le résultat d'une action et l'action elle-même ; c'est un concept qui s'entend dans le temps, se transforme minute après minute et il est irréversible. Il n'est donc pas facile de donner une définition «définitive» de la confiance. De nombreux auteurs en sciences sociales ont tenté de conceptualiser ce phénomène (Simmel, 1999 ; Origgi, 2007 ; Seligman, 2007). Pour Louis Quéré elle « est aujourd'hui en passe de devenir un sujet de controverse en sciences sociales » (Quéré, 2001, 9).

Chez les économistes, c'est un concept peu opératoire en dehors des procédures contractuelles (contrat signé par exemple); et pourtant l'actualité commerciale, économique ou managériale fait référence à ce concept: par exemple, on parle d'augmentation de niveau de confiance dans les achats sur internet (bon nombre d'internautes n'hésitent plus à donner leur numéro de carte bancaire); de refondation de la confiance suite à l'affaire Enron ${ }^{13}$ entre autres, affaire qui a conduit à une crise de la confiance dans les milieux financiers. Les économistes donnent donc une définition limitée au risque d'opportunisme (Williamson, 1975). Zucker (1986) distingue trois formes de confiance en fonction de leur mode de production :

- la confiance intuitu personae (characteristic based trust) qui est attachée à une personne en fonction de caractéristiques propres comme l'appartenance à une famille, une ethnie ou un groupe donné ;

12. Colloque « Du Mépris à la Confiance, de nouveaux comportements pour faire face à la complexité » organisé par l'Université de Technologie de Compiègne (TSH/IDTH), sous la direction de G. Le Cardinal et de J.F. Guyonnet en 1991.

13. Groupe américain de courtage en énergie qui a fait faillite alors qu'il jouissait de la confiance des actionnaires, qui faisaient aussi confiance au cabinet d'audit censé déceler les irrégularités financières et comptables. 
- la confiance relationnelle (process based trust) qui repose sur les échanges passés ou attendus en fonction de la réputation ou d'un don/contre don;

- la confiance institutionnelle (institutional based trust). Dans ce cas, la confiance est attachée à une structure formelle qui garantit les attributs spécifiques d'un individu ou d'une organisation.

Notre intérêt pour la confiance provient de ce qu'elle peut être mise au cœur du pilotage de projet et devenir un facteur qui change tout (Covey et al., 2008).

\section{La confiance comme levier de la coopération dans le projet SI}

Les projets sont en principe les lieux par essence de la coopération. Celle-ci se base autour de la communication et de la confiance entre les acteurs, les parties prenantes. La coopération est le propre même de l'entreprise car toute activité sociale, organisationnelle, requiert la coopération dès lors que l'individu seul ne parviendra pas au résultat. C'est l'action collective par laquelle les sujets contribuent aux mêmes résultats (De Terssac et al., 1996).

Selon Le Cardinal (Le Cardinal et al, 1996), le succès de la coopération entre les acteurs du projet implique des conditions :

- être en situation de vouloir et de pouvoir coopérer, c'est-à-dire se connecter physiquement, intellectuellement, psychologiquement aux autres; avoir le respect et la reconnaissance; être respecté dans son secret; exclure l'usage de la contrainte ou de la force ;

- reconnaitre les bénéfices des interactions en expérimentant l'intérêt de la coopération, en identifiant les apports réels de l'autre ;

- reconnaittre les difficultés de la coopération et aussi se demander si la coopération en valait la peine ;

- pouvoir distinguer les finalités de la coopération; dans certains cas de coopération, seul le résultat compte; dans d'autres un apprentissage mutuel de compétences se dessine et accroît la confiance; enfin un troisième type de finalité de la coopération est l'exploration des nouvelles idées, de nouveaux domaines, exploration impossible à faire tout seul.

Coopérer nécessite aussi bien la confiance que le contrat. En effet «tout se passe comme si le contrat, l'organigramme théorique, le travail prescrit étaient la partie visible de l'iceberg. La confiance interpersonnelle, l'organisation concrète et le travail réel en seraient les parties cachées, immergées ». (Le Cardinal et al, 1996, 74). Le contrat est nécessaire dans la coopération parce qu'il donne des garanties sur les conditions de réalisation de celle-ci. 
Le contrat assure la pérennité d'une activité de projet; les obligations, les limites, les interdits en sont définis en vue d'arriver aux résultats escomptés. Il est donc la partie écrite de la relation dans cette activité projet. Le cahier des charges de ce projet de SI est considéré comme la partie contractuelle.

Le contrat donne des garanties de coopération telles que l'équité, le libre engagement, etc. Mais est-ce vraiment suffisant pour coopérer efficacement ? La confiance est importante pour compléter ce contrat. L'expérience montre que même si les termes du contrat sont écrits, clairs, la réussite peut ne pas être au rendez-vous. Certes, la confiance n'exclut pas le contrôle souvent indispensable dans le projet, mais elle permettra d'une part de ne pas avoir à tout vérifier, par exemple (parce qu'on fait confiance) et d'autre part donne une dimension plus profonde et plus pérenne de la coopération.

Dans le cadre du projet, les relations entre les utilisateurs et les informaticiens d'une part, et les informaticiens entre eux d'autre part consistent souvent à rechercher en premier lieu s'il convient ou non de coopérer. Elle passera par la connaissance de l'autre, l'estimation de ses capacités techniques à réaliser le projet mais aussi à coopérer pour le mener à bien. Si cette démarche est accomplie par les différents partenaires, la confiance peut alors se concrétiser par un accord. Par exemple, s'accorder sur le contenu du cahier de charges : il revient aux informaticiens de traduire les besoins des utilisateurs dans un langage compréhensible par ces derniers (le langage des informaticiens est réputé souvent peu compréhensible).

\section{Comment amener les acteurs à coopérer?}

Les acteurs ou groupe d'acteurs qui communiquent développent dans le premier objectif de la communication (échange, circulation, transmission des informations) une vision commune ou des «représentations proches » qui seraient par exemple les langues, les valeurs, les idéologies, les expériences qui sont communes aux acteurs. La confiance dans la coopération se construit aussi par l'action. Cette action peut être par exemple de savoir compter sur l'autre et sa compétence, son efficacité, sa performance.

Comment arrive-t-on à la coopération ? Selon Le Cardinal, les sept étapes clés d'un projet doivent être respectées sinon la coopération en sera «perturbée »:

- la mise en présence qui va du coup de téléphone à la poignée de main pour la présentation des acteurs;

- la définition du projet commun, lieu où on découvre les enjeux, les engagements réciproques, les intentions, les sentiments cachés de l'autre ; 
- la qualification du projet, on se demande à ce stade si les ressources, les compétences, les savoir-faire sont disponibles, si toutes les tâches ont été identifiées ;

- la réalisation du projet, il s'agit de tenir compte pendant la réalisation du projet de tous les éléments internes et externes susceptibles de perturber la coopération (défaillance d'un acteur, risque lié au partenaire externe, etc.) ;

- l'évaluation des résultats est une cause d'échec de projet parce qu'elle porte sur le jugement de chaque acteur de ces résultats ; l'harmonisation des points de vue et des sentiments est gage d'une coopération future plus facile ;

- le partage des bénéfices ou des pertes, « les dividendes » d'un projet sont difficiles à partager parce que forcément inégalitaires; ces avantages ne sont pas seulement matériels, il y a par exemple, les connaissances acquises, pendant le projet, les outils, les relations nouées avec les autres acteurs, l'image de soi, des autres...

- la mise en absence, qui marque la façon de clôturer le projet ; la façon de se séparer à la fin du projet peut favoriser ou endommager le climat de confiance ; on célèbre la fin du projet en ayant un autre regard; et se révéler sous un autre jour favorisera une prochaine coopération.

Concernant ce projet de refonte de SI, nous avons focalisé notre démarche sur une méthode (Abba, 2007) dont le point de départ est la communication.

L'importance de la communication dans les projets n'est plus à démontrer. Même si elle ne règle pas tous les conflits, par exemple, elle est la base de la confiance puis de la coopération. Ces trois éléments constituent une trilogie qui va caractériser les dimensions humaines du projet :

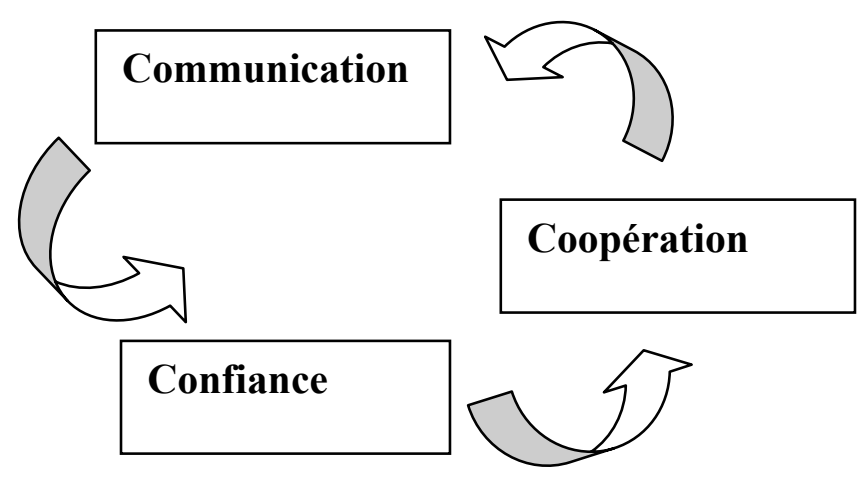

Figure 7. Processus trialogique de base 


\section{La méthode 4C : communication-confiance-coopération et créativité}

\section{Phase 1 : intégration bumaine}

Cette phase constitue, pour tout projet dans ses dimensions humaines, les préalables ou prérequis qui conduiront à asseoir une communication. Les acteurs du projet ont la même représentation du travail, parlent un langage commun. On s'assure en outre qu'ils ont les «bonnes informations", que les compétences individuelles et collectives sont en adéquation avec les tâches à accomplir et enfin que chaque acteur joue un rôle défini identifié.

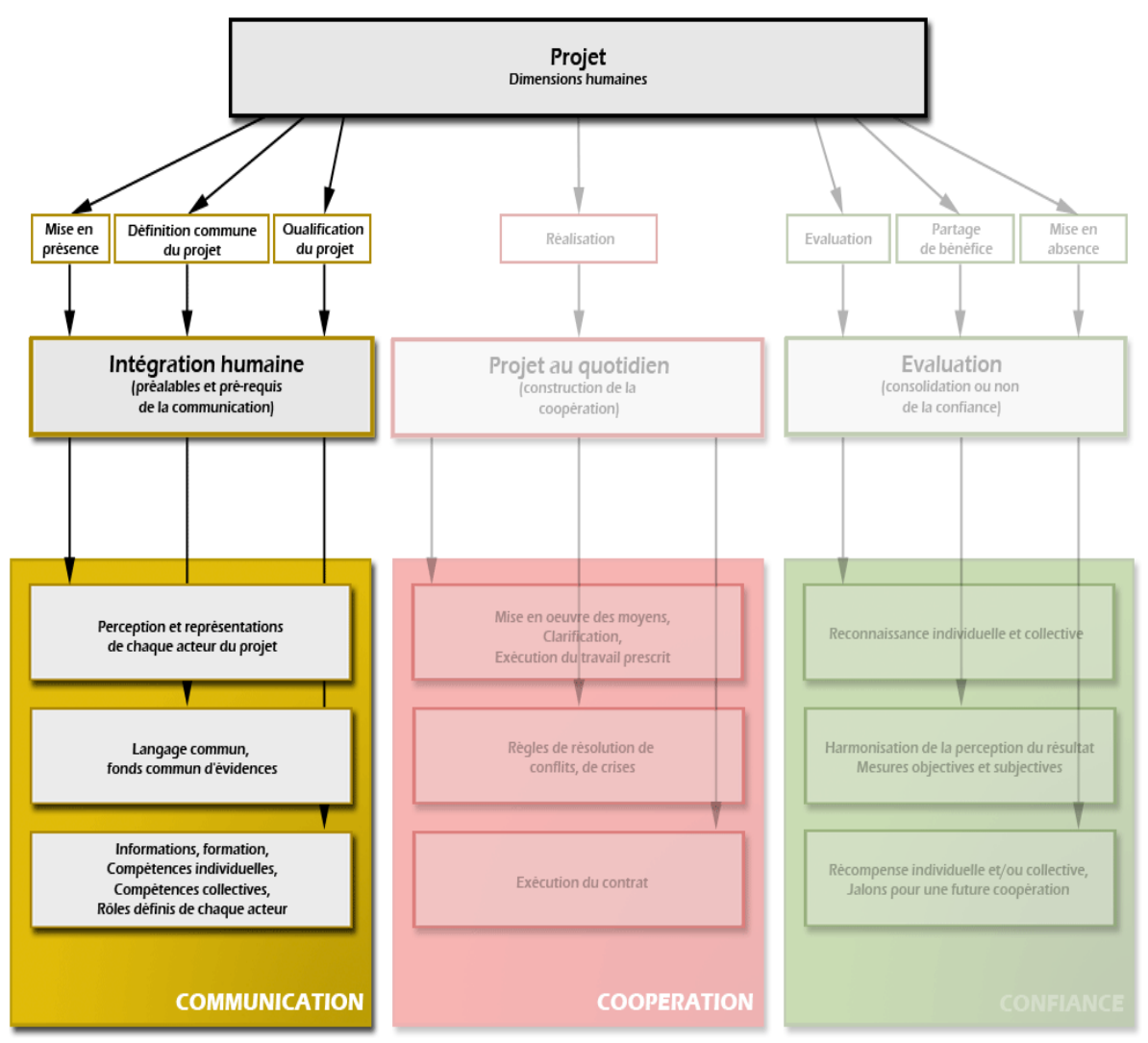

Figure 8. Construction du modèle : communication 


\section{Phase 2 : projet au quotidien et construction de la coopération}

Cette étape est celle de la construction de la coopération dans «l'usage » ou la vie du projet. Les acteurs font à la fois référence au contrat (la partie explicite ou écrite du projet) et posent les premières briques de la confiance. Des conflits peuvent surgir, mais les règles de résolution sont en place. Les moyens mis en œuvre permettent l'exécution du travail à faire. La construction de cette coopération est indissociable de l'étape précédente.

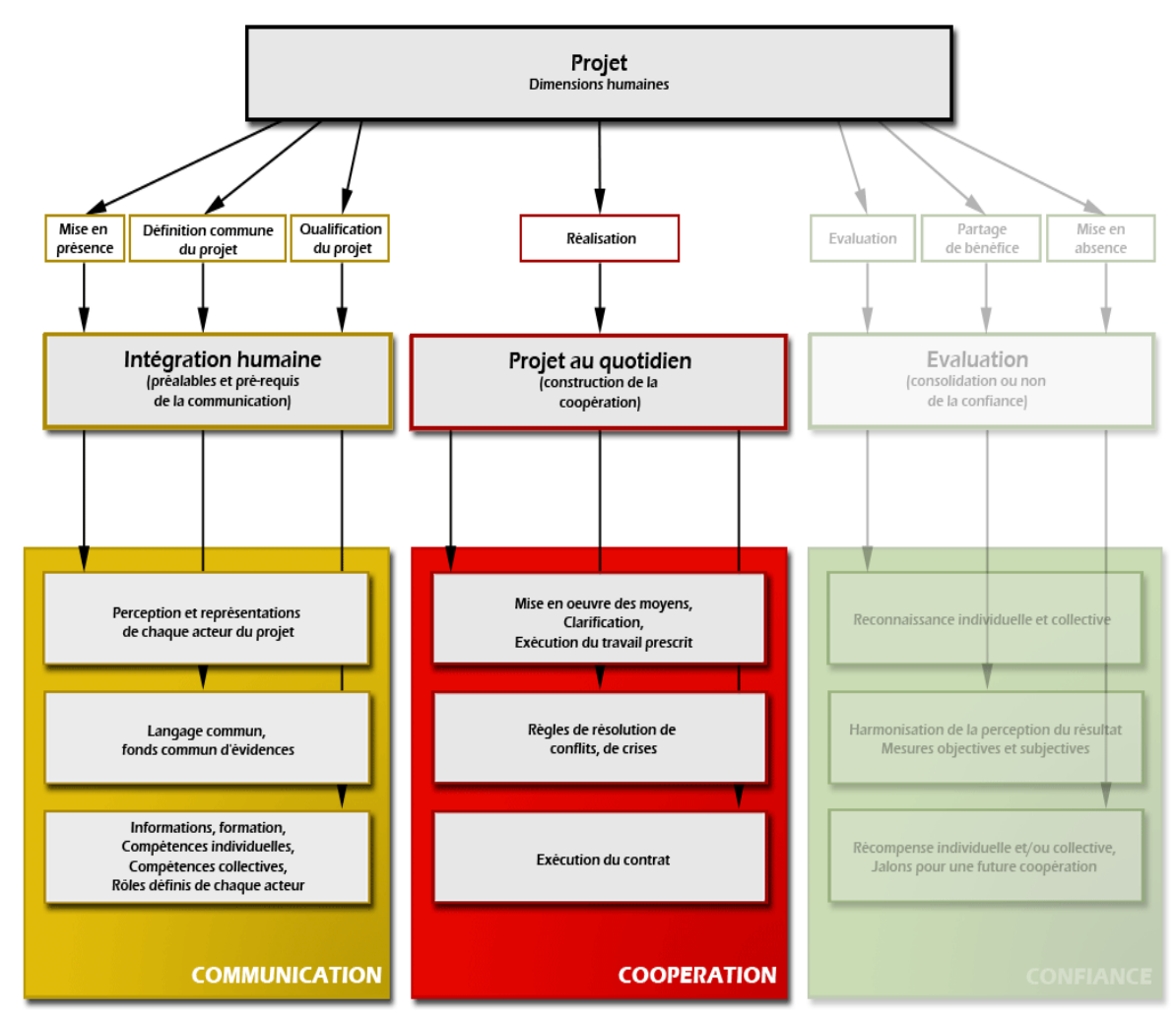

Figure 9. Construction du modèle : coopération

\section{Phase 3 : évaluation}

Cette phase est à la fois celle de l'issue du projet mais aussi consolide (ou non) la confiance créée tout au long des phases précédentes. La reconnaissance 
du travail des acteurs est fondamentale. Elle est différente de la récompense ou rétribution à l'issue du projet. Évaluer objectivement le travail n'est pas évident. À côté d'une mesure objective (objectifs atteints, produit ou service réalisé, etc.), il y a des dimensions subjectives de l'évaluation parce que chaque acteur a une liste de critères d'appréciation plus ou moins personnelle (en plus des critères qu'on peut appeler communs).

L'intérêt de l'évaluation est de poser les jalons d'une coopération future; pour ce faire, l'harmonisation des points de vue est nécessaire. Chaque acteur précise l'importance qu'il donne à ses critères.

Les bénéfices pour l'acteur dans cette phase peuvent être les connaissances et méthodes apprises, des nouvelles relations professionnelles, « les preuves » de ses compétences envers les autres, etc.

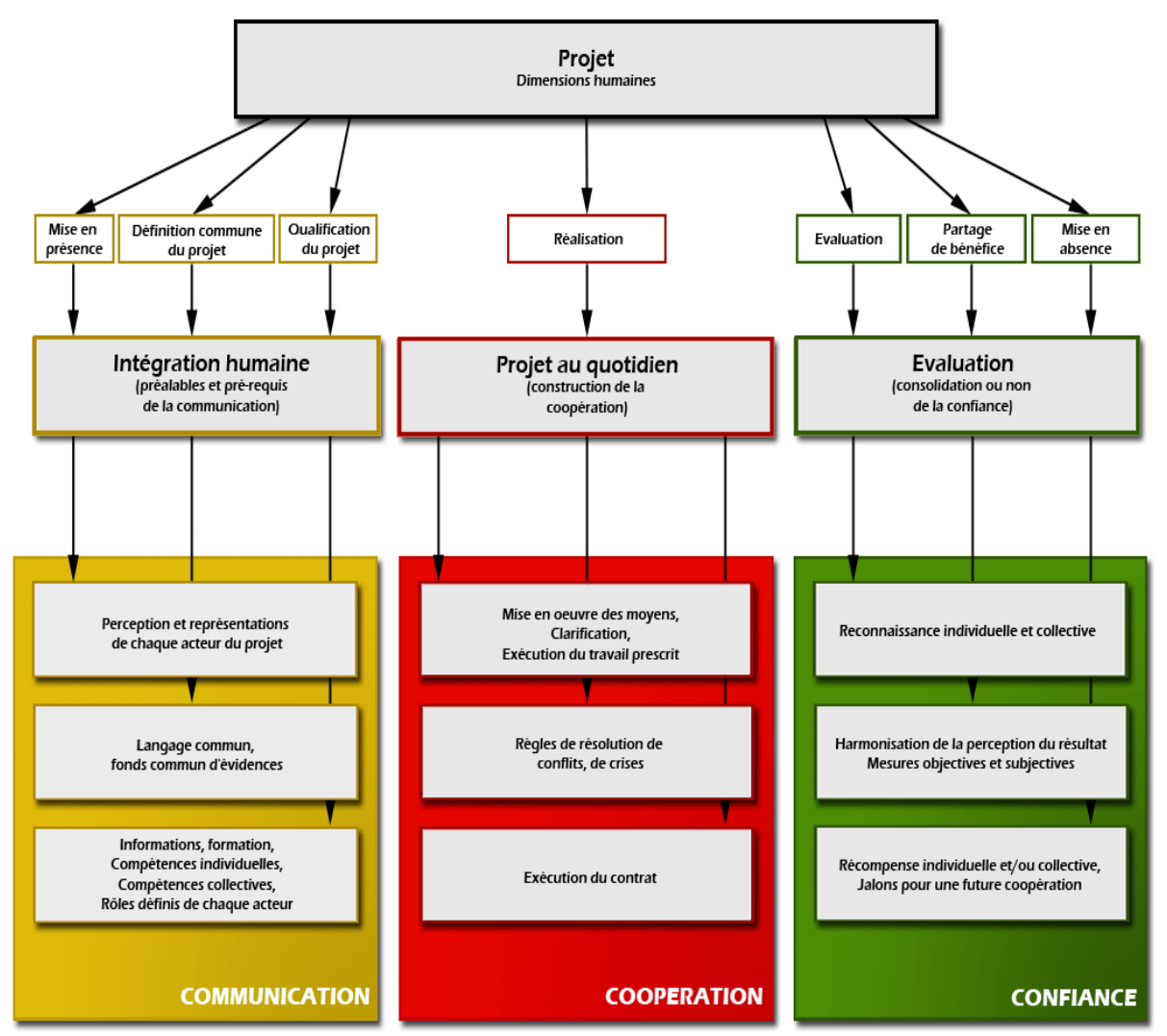

Figure 10. Construction du modèle : évaluation 


\section{Modèle général 4C}

Les trois phases nous conduisent à ce que nous appelons le modèle général 4C. Ces 3 phases du modèle ne sont pas assimilables aux étapes d'un projet tel que défini précédemment. Elles se complètent par un environnement organisationnel propice à la créativité individuelle et collective.

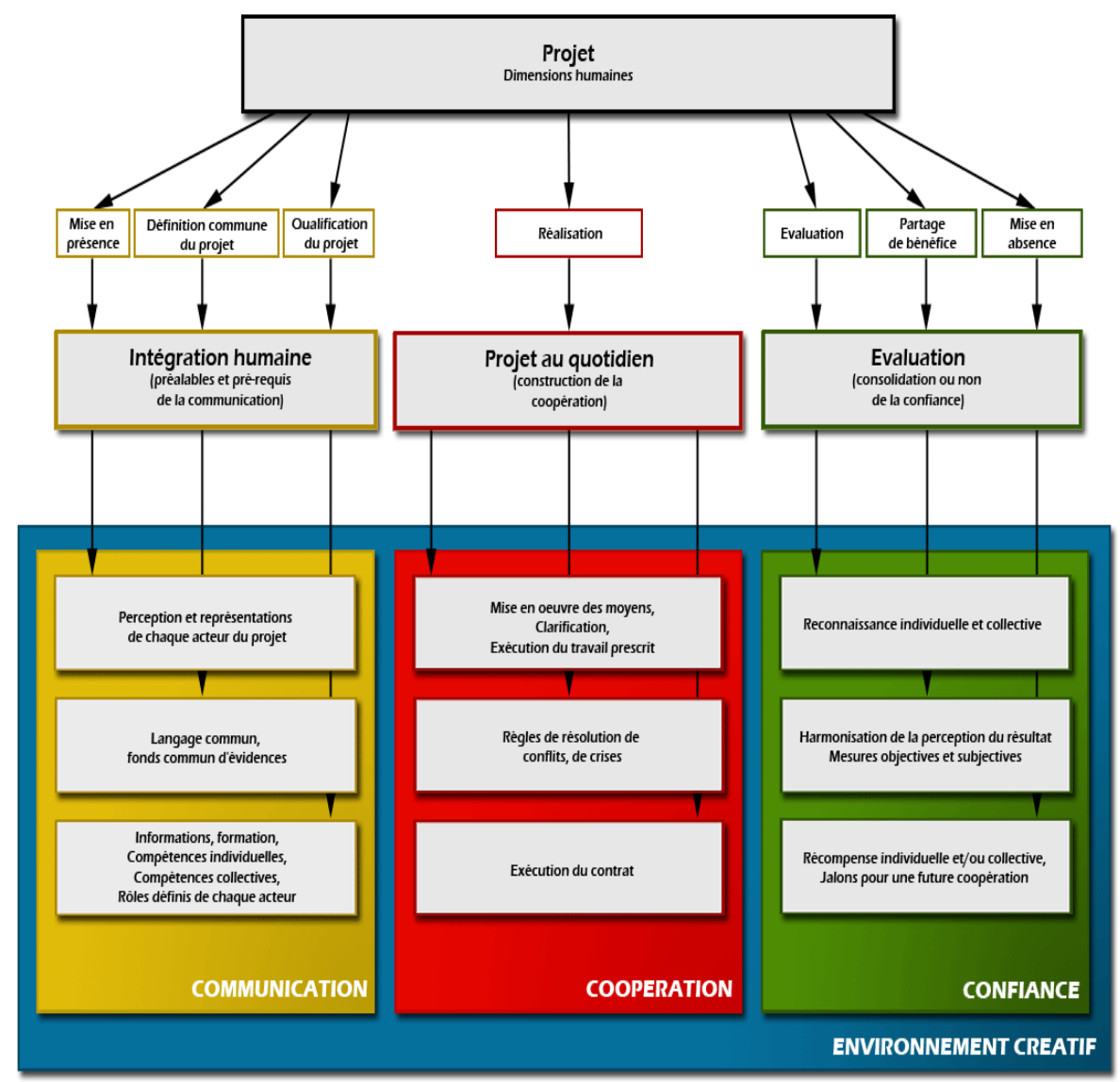

Figure 11. Modèle général 4C

\section{Déploiement du modèle dans le cadre du projet SI}

Les difficultés de communication dans le projet de refonte du SI ont été présentées notamment dans les observations des différentes situations entre acteurs. 


\section{De l'intégration au projet}

Dans le cas concret de mise en œuvre en entreprise, les premiers contacts consistent d'une part à «s'accepter» dans un projet en cours et, d'autre part, contribuent à desserrer les freins et blocages à l'origine des difficultés ambiantes.

Il n'existe pas de méthode ou méthodologie pour intégrer une équipe projet. On est généralement «adoubé » par la hiérarchie, ce qui n'est pas toujours sans conséquences sur l'équipe en place. La création d'un climat de confiance est la base de la future coopération. Comment alors passer de la méfiance perceptible à l'assurance, puis à la confiance ? Il est nécessaire de faire adhérer toutes les parties prenantes au projet. Les futurs usagers du système n'ont pas vraiment eu leur mot à dire. Il est donc impératif de remettre des «outils simples» de la communication. Les réunions avec les salariés, la publicisation dans le journal interne et l'intranet du groupe, le «mot» du DG ont servi de base. Communiquer sur quoi ? Les objectifs attendus et même l'après-projet. En effet certains acteurs ont demandé naturellement à être rassurés : cette technologie ne cacherait-elle pas un futur remplacement par la machine (les opératrices téléphoniques par exemple) ? Ces clarifications (a posteriori) ont été nécessaires dans un environnement projet caractérisé par des blocages. Quelle représentation les protagonistes du projet se faisaient-ils ?

Il n'est pas anodin de se poser cette question car comme le souligne A. Bouzon (2004, p. 129), « la représentation individuelle n'est pas la reproduction fidèle de la réalité mais une construction de celle-ci».

Les perceptions et représentations de ce projet informatique étaient clairement différentes selon les acteurs : les futurs usagers/clients du système étaient convaincus que la technologie ainsi apportée était un travail supplémentaire (de maitrise), passablement inutile, parce qu'ils estimaient avoir leur "propre quotidien»; l'équipe informatique (MOA/MOE) pensait quant à elle qu'une telle nouveauté ne pourrait qu'être bénéfique à l'entreprise.

À travers de multiples réunions et un accompagnement personnalisé, un langage commun s'est installé sur la vision du projet, ses objectifs. Cette émergence de fonds commun d'évidence a conduit à travailler de façon un peu plus communicative entre l'équipe informatique et les autres acteurs directement impactés par le projet.

Beaucoup de résultats de recherche indiquent que la formation est l'un des éléments les plus importants dans une exécution réussie de projet. La formation est souvent le lieu pour la communication de la nouvelle stratégie d'organisation, des nouvelles valeurs, des nouveaux outils, et des nouvelles manières d'effectuer le travail. Aux compétences métiers de chacun s'ajoutent des compétences à 
acquérir sur l'utilisation des outils notamment informatiques, sur lesquels sont formés ces acteurs : être capable de faire de la gestion documentaire (création, publication, archivage...) dans la base conçue à cet effet.

\section{De la construction de la coopération}

La durée un peu longue montre les freins auxquels ce projet a été confronté malgré les moyens mis en œuvre. Le premier travail a relevé de la simple ingénierie de projet qui consiste à revoir les phases et des jalons pour l'exécution du travail restant à faire. En effet, certaines années de la vie du projet n'ont pas été un exemple d'organisation. Cette apparente « désorganisation » a cristallisé les freins.

Il a fallu refonder les bases de la coopération entre l'équipe informatique et les autres acteurs du projet. Parmi ces derniers, certains éprouvaient des difficultés dans la maitrise de l'outil informatique et la prise en compte de ces difficultés par l'apport d'une aide (formation, accompagnement) fut perçue positivement. Les réunions programmées pour cet accompagnement constituent un terrain propice à «l'expression libre » sur le projet :

- estimation de la charge de travail restante en fonction de leur niveau d'appropriation de l'outil ;

- présentation des nouveautés et des fonctionnalités du système; etc.

La clarification des rôles de chacun dans le projet a participé aussi de cette construction de la coopération. L'instauration d'un dialogue permanent à travers des «forums sur l'informatique », questionnaire anonyme, a fait avancer le projet. C'est un lieu où la parole est «libérée » pour permettre aux collaborateurs de dire ce qu'ils pensent du projet, comment ils le vivent, la compréhension des tâches à effectuer (plan d'amélioration, indicateurs de performance, etc.)

\section{De l'évaluation du projet}

L'évaluation passe par la reconnaissance du travail de chacun. La particularité de ce projet est que cette phase n'a pas été spécialement mise en avant. L'upgrade du système a certes été obtenu à l'issue du projet mais les perceptions du résultat par les usagers du SI d'une part, l'équipe qualité et la maitrise d'ouvrage du projet (direction générale), d'autre part, ne sont pas les mêmes : les premiers ont le sentiment d'avoir "été forcés » au résultat et les seconds ainsi que la hiérarchie pensent qu'il s'agit là d'un succès éclatant! Objectivement, on peut mesurer le résultat obtenu puisque le système marche techniquement ; les indicateurs fonctionnent pour évaluer l'activité. 
Cependant il n'est pas possible de savoir quelles ont été pour les usagers/clients, les retombées non mesurables comme l'acquisition de nouvelles compétences, les nouvelles relations professionnelles, etc. Ce travail de démarche de progrès n'a pas été initié. Il n'est pas non plus possible d'évaluer pour ce projet si la confiance a été consolidée ou non et si des nouveaux jalons d'une future coopération ont été posés. Il s'agit d'une observation qui se fera sur le long terme.

\section{Axe d'amélioration de la méthode}

Le modèle n'est pas construit pour résoudre les conflits personnels (la démission d'un certain nombre d'acteurs en est la preuve). Dans ce cas, aucune communication ne saurait être d'une grande efficacité. En outre, la coopération relève de la volonté ou non de chaque acteur: on peut faire semblant de coopérer.

Une autre amélioration à apporter dans ce modèle est que la cognition individuelle n'a pas été suffisamment développée. La construction du projet est tributaire de la construction de l'identité et du sens par chaque acteur. Si les trois éléments de la cognition individuelle (information, représentation, évaluation) ont été mis en évidence dans ce modèle, trois autres (décision, émotion, action) demandent à être approfondis.

\section{Conclusion}

La place de la communication dans le projet, dès sa genèse, a été fondamentale pour conduire cette concertation puis construire la coopération entre les acteurs impliqués. Ces éléments ont constitué avec la confiance une «boucle récursive», une trilogie nécessaire pour créer un meilleur environnement de projet. De cet environnement nous avons fait l'hypothèse qu'il contribue d'une part à desserrer les freins naturellement inhérents à la composante humaine dès lors qu'il s'agit d'outils de changement organisationnel en particulier l'utilisation du numérique par le client (usager) de l'entreprise. Comme souvent, les outils de communication mis en œuvre, notamment le modèle 4C, n'ont pas pesé sur l'ingénierie et le pilotage de projet. Ce travail a contribué à montrer qu'il en est pourtant bien ainsi.

\section{Bibliographie}

Abba H., L'esprit 4C dans l'Entreprise : pour une coopération créative axée sur la communication et la confiance, Thèse de doctorat, Université de Valenciennes, 2007. 
$108 \mathrm{LCN} \mathrm{n}^{\circ}$ 4/2010. Piloter l'entreprise à l'ère du numérique

Alter S., "A general yet useful theory on Information Systems", Communications of the association for information systems, vol. 1, $\mathrm{n}^{\circ} 13,2000$.

Argyris C., Savoir pour agir. Surmonter mes obstacles de l'apprentissage organisationnel, (Trad. Fr) Paris InterEditions, 1995.

Bagla-Gökalp L., Sociologie des organisations Paris, La Découverte, 2003.

Bouchikhi H., La structuration des organisations - concepts constructivistes et étude de cas », Paris, Economica, 1990.

Boutinet J-P., Psychologie des conduites à projet, Paris, PUF, 2006

Bouzon A., La place de la communication dans la conception de systèmes à risques, Paris, L'Harmattan, 2004.

Bouzon A., (Coord.) Technologies de l'information et de la communication dans les organisations, théories et Pratiques, Octarès, Toulouse, 2005.

Cleland D.I., King W.R., L'Analyse de systèmes technique avancée de management, Éditions Entreprise moderne, Paris, 1970.

Covey S.R., Merill R.R., Roche D. (trad. Fr), Le pouvoir de la confiance : Le facteur qui change tout, Paris, Editions Générales, 2008.

Crozier M., Friedberg E., L'acteur et le système : les contraintes de l'action collective, Paris, Seuil, 1977.

De Terssac G., Maggi B., «Autonomie et conception », Coopération et conception, Octarès, Toulouse 1996, p. 243-266.

Dorrer L., Hommes et projets informatiques : dix commandements pour réussir, Paris, HermèsLavoisier, 2004.

Drucker P., Au-delà du capitalisme la métamorphose de cette fin de siècle, Paris, Dunod, 1993.

Durand A., Huart J., Leleu-Merviel S., «Vers un modèle de programme pour la conception de document », Revue internationale Hypertextes, Hypermédia, 1(1),79-101.

Ein-Dor P., Segev E., "A classification of Information Systems", Information System Research, vol. 4, n², 1993, p. 166-204.

Fayol H., "Administration industrielle et générale» Bulletin de la Société de l'Industrie Minérale, $\mathrm{n}^{\circ} 10,1916$, p. 5-164, Paris, Dunod.

Feeny D., Willcoks L. «Bien gérer la sous-traitance », L'art du management de l'information, Village mondial, Paris, 2000, p. 66-69.

Germain M., Management des nouvelles technologies et e-transformation regard systémique sur les TIC dans les organisations, Paris, Economica, 2006.

Getz I., «Systèmes d'information : l'apport de la psychologie cognitive », Revue Française de gestion, $\mathrm{n}^{\circ} 99$, p. 92-108, 1994. 
Giddens A., La construction de la modernité, Paris, PUF, 1987.

Guyot B., «Eléments pour une approche informationnelle dans les organisations », Sciences de la société, $\mathrm{n}^{\circ}$ 63, 2004, p. 11-25.

Hirscheim R., Klein H., Lyytinen K., Information systems development and data modelling: conceptual and philosophical foundations, Cambridge University Press, 1995.

Huart J., Kolski C., Leleu-Merviel S., «Problème de création en multimédia : marier l'expérience de l'audiovisuel et la rigueur de la qualité », indexé Google Scholar, Cabiers du CIRCAV, 12:31-49, 2000.

Kappelman L.A., McLean E.R., "User Engagement”, The Development, Implementation and use of Information Technologies, Proceedings of the 27th HICSS, Maui, Hawaii, 1994, p. 512-521.

Le Cardinal G., Pouzoullic B., Guyonnet F., La dynamique de la confiance, Paris, Dunod, 1996.

Le Goff J.P., Les illusions du management, pour le retour du bon sens, La Découverte, Paris, 2000.

Leleu-Merviel S., Objectiver l'humain, Paris, Hermès-Lavoisier, 2008.

Leleu-Merviel S., La conception en communication, Paris, Hermès sciences publications, 1997.

Leleu-Merviel S., Useille P., "Quelques révisions du concepts d'information», in Problématiques émergentes dans les sciences de l'information, F. Papy (Ed.) HermèsLavoisier, 2008.

Le Moigne J.L., «Du Mépris à la Confiance, de nouveaux comportements pour faire face à la complexité », Colloque organisé par l'Université de Technologie de Compiègne (TSH/IDTH), sous la dir. de G. Le Cardinal et de J.F. Guyonnet, 1991.

Lewin K., Frontiers in Group Dynamics, 1946.

Luhmann N., La confiance, un mécanisme de réduction de la complexité sociale, Paris, Economica, 2006.

Marciniak, R., Rowe F., Systèmes d'information et dynamique d'organisation, Paris Economica, 2005.

March J., Cyert R., A behavioral theory of firm, Englewood Cliffs, Prentice-Hall inc. (1963).

Markus L., "Les salariés face aux technologies », in L'art du management de l'information, Paris, Village mondial, 2000, p. 222-226.

Maslow A., Devenir le meilleur de soi-même. Besoins fondamentaux, motivation et personnalité (Trad. Fr.), Paris, Eyrolles, 2008.

Mason R., Mitroff I., "A program for research on management Information Systems”, in Management Science, vol. 19, $n^{\circ}$ 8, p.475-487, 1973.

Mélèse J., Approche systémiques des organisations : vers l'entreprise à complexité humaine, Paris, Éditions de l'organisation, 1990. 
$110 \mathrm{LCN} \mathrm{n}{ }^{\circ}$ 4/2010. Piloter l'entreprise à l'ère du numérique

Mucchielli A., Études des communications : approche par modélisation des relations, Paris, A. Colin, 2004.

Morley C., Hugues J., Leblanc B., UML pour l'analyse d'un système d'information, Paris, Dunod, 2002.

Origgi G., Qu'est-ce que la confiance? Paris, Vrin, 2007.

Perret V., «La gestion du changement organisationnel : articulation de représentations ambivalentes » $5^{\circ}$ Conférence Internationale de management stratégique, $\mathrm{n}^{\circ}$ 54, Lille, 1996.

Quan D., L'impossible conduite d'un projet de système d'information, Paris, Hermès-Lavoisier, 2006.

Quéré L., Présentation, Réseaux, 2001/4, n¹08, p. 9-12.

Reix R., Systèmes d’information et management des organisations, Paris, Vuibert, 2005, 5éd.

Reynaud A., Petitbon F., Heckmann H., Restaurer la confiance dans l'entreprise- renouveler le lien entre employeur et collaborateurs, Paris, Dunod, 2010.

Roux A., "système d'information et dispositif informationnel : quelle articulation ? ", in Sciences de la société $\mathrm{N}^{\circ} 63.2004$, p. 27-39.

Rowe F., Faire de la recherche en systèmes d'information, Paris, Vuibert, 2002.

Sainsaulieu R., Sociologie de l'organisation et de l'entreprise, Paris, Presses de la Fondation Nationale des sciences politiques \& Dalloz, 1987.

Seguin, F., Chanlat, J-F., L'Analyse des organisations une anthologie sociologique : les théories des organisations, Tome 1, Montréal, éd. G. Morin, 1986.

Seligman A., The problem of Trust, Princeton, Princeton University Press, 1997.

Simmel G., Philosophie de l'argent, Paris, PUF, 1999.

Simon H., Administration et processus de décision, (trad. Fr) Paris, Économica, 1983.

Simon H., "Theories of Decision-Making in Economics and Behavioral Science", American Economic Review, 49, n 1, p. 253-283, 1959.

Taylor F.W., Principes d'organisation scientifique des usines, (trad. Fr), Paris, Dunod, 1912.

Volle M., Chelli H., Urbaniser l'entreprise et son système d'information : Guide des entreprises agiles, Paris, Vuibert, 2003.

Weber M., Economie et société 2. L’Organisation et les puissances de la société dans leur rapport avec l'économie, (trad. Fr), Paris, Pocket 1995.

Williamson O.E., Markets and Hierarchies: Analysis and Antitrust Implications, Free Press, 1975.

Zucker L., "The production of trust", Research in Organizational behaviour, 1986, vol. 8, p. 53-111. 